\title{
INTERFERÊNCIA INTERESPECÍFICA ENTRE AMARANTHUS HYBRIDUS L. E AMARANTHUS VIRIDIS L. ${ }^{1}$
}

\begin{abstract}
ANGELA MARIA MALUF²
RESUMO - O objetivo deste trabalho foi estudar a interferência interespecífica entre duas espécies de Amaranthus - A. viridis e A. hybridus --, esta última com dois biótipos distintos (verde e roxo); ambas ocorriam associadas em dois locais. Nas populações de cada local foram feitos ensaios de substituição independentes, em vasos, nos quais observou-se que as populações que germinam mais prontamente são as que vencem em competição, ou seja, se estabelecem e produzem maior número de sementes. Houve tendência de $A$. hybridus (tipo verde) dominar tanto A. hybridus (tipo roxo) como A. viridis, e de $A$. hybridus (tipo roxo) dominar $A$. viridis. Em cultivo misto, houve casos em que as espécies estavam competindo pelos mesmos recursos, ou explorando recursos diferentes do ambiente, ou mesmo com antagonismo mútuo.
\end{abstract}

Termos para indexação: alocação de energia, alocação de biomassa, planta infestante, amaranto, ensaios de substituição.

\section{INTERSPECIFIC INTERFERENCE BETWEEN AMARANTHUS HYBRIDUS L. AND AMARANTHUS VIRIDIS L.}

\begin{abstract}
The objective of this paper was to study the interspecific interference between two Amaranthus species - A. viridis and A. hybridus -, the latter with two distinct biotypes (green and purple); both species ocurred in two localities. In the populations of each of the locations, some independent substitution trials were made in pots, and it could be concluded that the populations that germinate more readily are those which are superior in competition i.e., those that establish themselves and produce the largest number of seeds. There was a tendency for A. hybridus (green biotype) to dominate both $A$. hybridus (purple biotype) and A. viridis, and for A. hybridus (purple biotype) to dominate $A$. viridis. In mixed stands, there were cases where the species were competing for the same resources, cases where they were exploiting different environmental resources, and even cases of mutual antagonism.
\end{abstract}

Index terms: energy allocation, biomass allocation, weed, amaranth, substitution trials.

\section{INTRODUÇÃ̃O}

Para que ocorra competição, há necessidade de sobreposição suficiente dos nichos dos indivíduos que competem, de modo que eles utilizem os mesmos recursos.

Quando, em circunstâncias competitivas, os danos que uma espécie causa nela própria é maior que o dano que ela causa na outra espécie, elas podem coexistir. Sendo assim, espécies que são com-

\footnotetext{
${ }^{1}$ Aceito para publicação em 30 de setembro de 1998 .

${ }^{2}$ Enge Agrạ, Drạ, Instituto de Botânica, Caixa Postal 4005, CEP 01061-970 São Paulo, SP. E-mail: amaluf@smtp-gw.ibot.sp.gov.br
}

petidoras completas não podem coexistir indefinidamente, de acordo com o princípio de Gause ou princípio de exclusão competitiva (McNaughton \& Wolf, 1973).

As interações positivas e negativas entre as espécies em comunidades vegetais determinam o sucesso da invasão (Caldwell et al., 1996; Thébaud et al., 1996; Callaway \& Walker, 1997).

Segundo Bertness \& Callaway, citados por Holmgren et al. (1997), quando a associação se desenvolve na parte mais produtiva do gradiente ambiental ocorre a competição, enquanto em condições adversas, a facilitação é mais importante.

No gênero Amaranthus, alguns autores estudaram os diversos aspectos da competição 
interespecífica como competição por luz e por solo (Schreiber, 1967), relação da habilidade competitiva com a de fixar $\mathrm{CO}_{2}$ (Black et al., 1969) e arquitetura de planta (Vargas, 1985).

Em plantas invasoras, pode-se estudar a agressividade de uma espécie em relação à outra por experimentos de substituição ou experimentos em séries repassadas, proposto por DeWit, citado por Harper (1977) e utilizado por Vargas (1985), em Amaranthus, cuja freqüência relativa dos competidores varia, porém a densidade permanece constante.

As relações mútuas das duas espécies em referência à demanda de recursos podem ser expressas pela produtividade total relativa (PTR), que é o somatório das produtividades relativas (PR) das duas espécies, sendo a $\mathrm{PR}$ equivalente à produtividade da espécie na mistura em relação à produtividade $\mathrm{da}$ mesma espécie em estande puro. $\mathrm{O}$ valor $\mathrm{PTR}=1,0$ significa que as duas espécies estão demandando os mesmos recursos limitados do ambiente; o valor de PTR $>1,0$ sugere que as espécies demandam recursos diferentes do ambiente, evitando competição entre elas e/ou mostrando alguma forma de relação simbiótica; e PTR < 1,0 implica antagonismo mútuo (Harper, 1977). Essa proposta de se estudar a associação de duas espécies em mistura através da PR e PTR foi feita por DeWit \& Van Den Bergh (1965).

Com o objetivo de entender o tipo de associação de espécies comumente encontradas no gênero Amaranthus e o sucesso de seu estabelecimento, este trabalho investigou a interferência interespecífica em duas espécies, $A$. viridis e $A$. hybridus, esta última com dois biótipos distintos, tipo verde e tipo roxo.

\section{MATERIAL E MÉTODOS}

Foram utilizadas sementes de duas espécies de Amaranthus que ocorriam associadas, A. viridis L. e A. hybridus L., esta última com dois biótipos distintos. As identificações taxonômicas foram realizadas por especialistas do Instituto de Botânica (São Paulo, SP) e da UNESP (Rio Claro, SP). As sementes das espécies foram coletadas em dois locais: no local 1 (campo de milho), $A$. hybridus (tipo verde) ocorria associado a $A$. viridis, e no local 2 (campo de leguminosas forrageiras), além desses dois competidores, ocorria o biótipo A. hybridus (tipo roxo). É interessante mencionar que, no ano anterior a essa coleta, nos dois locais ocorriam os biótipos roxo e verde de $A$. hybridus, além das espécies $A$. retroflexus e A. spinosus.

Após a coleta de panículas contendo sementes maduras, de cerca de 30 plantas, essas foram colocadas em sacos de papel e secadas ao sol e/ou em estufa com circulação forçada de ar a $27-28^{\circ} \mathrm{C}$, até a deiscência dos frutos. O beneficiamento das sementes foi realizado pela fricção dos frutos num recipiente, seguida de agitação para a separação das sementes das estruturas dos frutos.

As sementes de todos os indivíduos da população foram misturadas para a instalação de quatro ensaios de substituição, em casa de vegetação, para se medir a agressividade de uma espécie em relação à outra (DeWit, citado por Harper, 1977).

Para facilidade de identificação dos materais, cada um deles será denominado por uma sigla (iniciais do gênero e espécie), que se refere à espécie, e por um número que se refere ao local de coleta. No caso da espécie $A$. hybridus, a sigla conterá ainda a letra $\mathrm{v}$ ou $\mathrm{r}$, indicando o biótipo verde ou roxo, respectivamente. Ainda, eles serão designados, no decorrer do trabalho, por populações, uma vez que este termo engloba comparações entre espécies diferentes e entre os biótipos verde e roxo de $A$. hybridus.

Nas duas espécies do local 1, foi instalado o ensaio Ahv-1:Av-1, e nas duas espécies (três biótipos) do local 2, foram instalados outros três ensaios: Ahv-2:Av-2, Ahv-2:Ahr-2 e Av-2:Ahr-2.

Convém observar que o caso do ensaio envolvendo Ahv-2:Ahr-2, na realidade, é uma competição intrabiotípica e, portanto, intraespecífica. No entanto, para designar qualquer ensaio de substituição, no decorrer do trabalho, será utilizado o termo competição interespecífica.

Foram utilizadas cinco proporções de sementes das duas espécies $(0: 24 ; 6: 18 ; 12: 12 ; 18: 6 ; 24: 0)$ e não foi feito o desbaste de plântulas; a competição partiu do estádio de sementes.

As sementes das duas espécies foram misturadas (total de 24 sementes - $\mathrm{D}_{\mathrm{o}}$ ) e semeadas na superfície de vasos de 0,5 litro de capacidade, contendo substrato hortícola produzido pela Mogiana Alimentos S.A., que é constituído basicamente de material orgânico, de fonte não identificada. As regas foram realizadas conforme a necessidade, mantendo o substrato úmido. A densidade de 24 sementes por vaso foi determinada pela capacidade de suporte do ambiente (dimensões dos vasos), através de ensaios preliminares.

Foram determinados o número de plantas 10 dias $\left(\mathrm{N}_{10}\right)$ e 20 dias $\left(\mathrm{N}_{20}\right)$ após a semeadura, e na época do desmonte das parcelas, foram contados: número total de 
plantas por vaso (NTP); de plantas com sementes por vaso (NPS) e de plantas com sementes e flores, ou somente flores, por vaso (NPSF). Nessa ocasião foi medida a altura de planta (AP), em centímetros, do colo da planta até a inserção da última folha para as plantas que não haviam entrado em fase reprodutiva, ou até o ápice da inflorescência principal, para as plantas que estavam em fase reprodutiva, e determinado o número de folhas (NF), contando o número de folhas ou de cicatrizes foliares existentes no caule principal. As folhas mais jovens com menos de um centímetro, quando as plantas estavam com inflorescência, não foram consideradas. Foi estabelecido, ainda, aos 60 dias, o estádio de desenvolvimento das plantas (ED), seguindo um sistema de notas, onde nota $1=$ plantas apenas em fase vegetativa, $2=$ plantas em fase reprodutiva, porém sem sementes maduras, $3=$ plantas em fase reprodutiva, com pelo menos uma semente madura. Em seguida , foram calculados: índice de espécie total (IET) - com relação a todas as plantas presentes no vaso, definiu-se o valor +1 para cada uma das plantas de uma espécie e o valor -1 para as plantas da outra espécie, e calculou-se o IET pela soma dos valores de cada planta, dividido pelo número total de plantas; índice de espécies com sementes (IES) - fez-se o mesmo cálculo anterior, porém apenas com as plantas que tinham pelo menos uma semente madura, sendo o divisor do somatório o número de plantas com sementes; e índice de espécie com sementes e flores ou somente flores (IESF) - fêz-se o mesmo cálculo anterior, porém, atribuíram-se os valores das plantas que tinham entrado em fase reprodutiva, ou seja, com flores e sementes ou somente flores. Foram atribuídos os valores +1 ou -1 às espécies, nos diferentes ensaios de substituíção, da seguinte forma: $\operatorname{Ahv}-1(+1): \operatorname{Av}-(-1) ; \operatorname{Ahv}-2(+1): \operatorname{Av}-2(-1)$; Ahv-2(+1):Ahr-2(-1); Av-2(+1):Ahr-2(-1).

Após as determinações já mencionadas, as plantas foram colocadas em sacos de papel e secadas em estufa com circulação forçada de ar, a $75^{\circ} \mathrm{C}$, até peso constante. Em seguida, foi quantificada a distribuição da biomassa, pela obtenção do peso de matéria seca das diferentes partes da planta, de acordo com o método descrito em Harper \& Ogden (1970). Foram determinados o peso de matéria seca, em gramas, de folhas $\left(\mathrm{V}_{1}\right)$; de ramos + caules $\left(\mathrm{V}_{2}\right)$; de folhas + ramos + caule, ou seja, da biomassa vegetativa da parte aérea (V); de inflorescências + sementes, ou seja, da biomassa reprodutiva $(\mathrm{R})$; e a produção total por vaso $(\mathrm{PT}=\mathrm{R}+\mathrm{V})$.

Para minimizar os efeitos das diferenças de luminosidade, na casa de vegetação, foi utilizado o delineamento em blocos casualizados, com quatro repeti- ções. Cada experimento constou de 20 parcelas experimentais.

Com os dados de $V_{1}, V_{2}$, R e PT, foram calculadas as produtividades relativas de cada espécie - PR e a produtividade total relativa - PTR (DeWit \& Van Den Bergh, 1965). Nos estandes puros (0:24 e 24:0) considerou-se PTR $=1$, situação na qual as plantas demandam os mesmos recursos do ambiente.

As contagens dos números de plantas: $\mathrm{N}_{10}, \mathrm{~N}_{20}, \mathrm{NPT}$, NPS, NPSF foram transformadas em porcentagem da densidade inicial de semeadura $\left(D_{0}=24\right.$ sementes por vaso $)$ e as produções de matéria seca, $V_{1}, V_{2}, V$ e $R$, foram transformadas em porcentagem da produção total (PT), para se obter a porcentagem de alocação de biomassa de cada parte da planta.

Todos os dados foram interpretados apenas em termos de média, a não ser os de produção, nos quais foram feitas as análises de variância em blocos ao acaso, e os testes de Tukey, para comparação de médias, e calculados os coeficientes de variação experimental (CV\%), de acordo com Pimentel-Gomes (1981). Foram realizadas todas as correlações simples entre as características analisadas (Steel \& Torrie, 1980).

\section{RESULTADOS E DISCUSSÃO}

Houve variação no ciclo de vida das espécies conforme o ensaio, de modo que o desmonte das parcelas ocorreu 57 dias após a semeadura nos ensaios Ahv-1:Av-1, Ahv-2:Av-2, Ahv-2:Ahr-2 e aos 63 dias no Av-2:Ahr-2.

No ensaio de substituição entre as populações do local 1 (Ahv-1:Av-1), as tendências de maiores alturas de planta (AP) foram encontradas nas proporções 6:18 e 12:12, o que mostra que nos cultivos mistos, onde Av-1 participa com $50 \%$ a $75 \%$ da densidade, são os que produzem a maior altura da planta. O número de folhas (NF) e o estádio de desenvolvimento (ED) não apresentaram tendência marcante (Tabela 1).

Nas populações do local 2, as respostas foram bem diferentes. No ensaio de Ahv-2:Av-2, de modo geral, as médias mostram que a altura da planta, o número de folhas e o estádio de desenvolvimento foram menores, na proporção 0:24 do que nas demais, onde o Ahv-2 entra em alguma proporção (Tabela 1). 
No caso do ensaio Ahv-2:Ahr-2 (Tabela 1), as maiores médias de altura da planta e número de folhas foram encontradas na proporção 12:12. Porém, o maior estádio de desenvolvimento foi encontrado na proporção 24:0. Ainda, comparando-se as proporções 0:24 e 24:0, observa-se que o Ahv-2 (24-0) tem maiores valores destas características que o Ahr-2 (0-24), o que indica que o Ahv-2 cresceu mais, e que maior número de plantas deste biótipo conseguiu chegar à fase reprodutiva durante o período experimental; o biótipo roxo tem um ciclo de vida mais longo.

TABELA 1. Médias da altura de planta, em $\mathrm{cm}$ (AP), número de folhas (NF) e estádio de desenvolvimento (ED) de Amaranthus, em quatro ensaios de substituição independentes envolvendo as duas populações do local 1 (Ahv-1:Av-1) e as três populações do local 2 (Ahv-2:Av-2; Ahv-2:Ahr-2; Av-2:Ahr-2).

\begin{tabular}{lrrr}
\hline Proporções $^{1}$ & AP & NF & ED \\
\hline Ahv-1:Av-1 & & & \\
$0: 24$ & 27,65 & 8,74 & 2,13 \\
$6: 18$ & 34,64 & 10,63 & 2,01 \\
$12: 12$ & 35,46 & 11,01 & 1,96 \\
$18: 6$ & 24,22 & 9,08 & 1,72 \\
$24: 0$ & 26,36 & 10,23 & 2,00 \\
Ahv-2:Av-2 & & & \\
$0: 24$ & 16,53 & 8,06 & 1,60 \\
$6: 18$ & 26,93 & 9,82 & 2,10 \\
$12: 12$ & 30,41 & 10,80 & 2,21 \\
$18: 6$ & 32,23 & 11,30 & 2,25 \\
$24: 0$ & 29,80 & 12,14 & 2,26 \\
Ahv-2:Ahr-2 & & & \\
$0: 24$ & 22,09 & 9,86 & 1,36 \\
$6: 18$ & 25,76 & 11,18 & 1,71 \\
$12: 12$ & 37,35 & 12,75 & 1,79 \\
$18: 6$ & 26,93 & 10,72 & 1,87 \\
$24: 0$ & 31,33 & 11,03 & 2,30 \\
Av-2:Ahr-2 & & & \\
$0: 24$ & 33,26 & 14,17 & 1,77 \\
$6: 18$ & 38,64 & 14,04 & 1,67 \\
$12: 12$ & 20,93 & 9,91 & 1,46 \\
$18: 6$ & 17,40 & 8,84 & 1,36 \\
$24: 0$ & 19,81 & 8,09 & 1,61 \\
\hline
\end{tabular}

${ }^{1}$ Ahv-1: Amaranthus hybridus - tipo verde, do local 1; Av-1: A. viridis, do local 1; Ahv-2: A. hybridus - tipo verde, do local 2; Ahr-2: A. hybridus - tipo roxo, do local 2; Av-2: A. viridis, do local 2.
Para o ensaio de substituição Av-2:Ahr-2, a proporção 0:24 foi superior a 24:0 quanto à altura da planta e quanto ao estádio de desenvolvimento, cujas médias foram decrescendo progressivamente à medida que se aumentava a proporção de Av-2, o que indica que Ahr-2 se desenvolve mais que o Av-2 (Tabela 1).

A Tabela 2 apresenta os índices de espécie total (IET), de espécie com sementes (IES) e de espécie

TABELA 2. Índice de espécie total (IET), índice de espécie com sementes (IES) e índice de espécie com sementes e flores ou somente flores (IESF), de Amaranthus, em quatro ensaios de substituição independentes, envolvendo as duas populações do local 1 (Ahv-1:Av-1) e as três populações do local 2 (Ahv-2:Av-2; Ahv-2:Ahr-2; Av-2:Ahr-2).

\begin{tabular}{|c|c|c|c|}
\hline Proporções $^{1}$ & IET & IES & IESF \\
\hline \multicolumn{4}{|l|}{ Ahv-1:Av-1 } \\
\hline $0: 24$ & $-1,00$ & $-1,00$ & $-1,00$ \\
\hline $6: 18$ & $+0,45$ & $+0,56$ & $+0,51$ \\
\hline $12: 12$ & $+0,84$ & $+0,88$ & $+0,78$ \\
\hline $18: 6$ & $+1,00$ & $+1,00$ & $+1,00$ \\
\hline $24: 0$ & $+1,00$ & $+1,00$ & $+1,00$ \\
\hline \multicolumn{4}{|l|}{ Ahv-2:Av-2 } \\
\hline $0: 24$ & $-1,00$ & $-0,75$ & $-1,00$ \\
\hline $6: 18$ & $-0,64$ & $-0,39$ & $-0,41$ \\
\hline $12: 12$ & $-0,03$ & $+0,04$ & $+0,11$ \\
\hline $18: 6$ & $+0,89$ & $+1,00$ & $+1,00$ \\
\hline $24: 0$ & $+1,00$ & $+1,00$ & $+1,00$ \\
\hline \multicolumn{4}{|l|}{ Ahv-2:Ahr-2 } \\
\hline $0: 24$ & $-1,00$ & $-0,75$ & $-1,00$ \\
\hline $6: 18$ & $-0,31$ & $-0,16$ & $+0,38$ \\
\hline $12: 12$ & $+0,32$ & $+0,13$ & $+0,59$ \\
\hline $18: 6$ & $+0,61$ & $+1,00$ & $+0,80$ \\
\hline 24:0 & $+1,00$ & $+1,00$ & $+1,00$ \\
\hline \multicolumn{4}{|l|}{ Av-2:Ahr-2 } \\
\hline $0: 24$ & $-1,00$ & $-1,00$ & $-1,00$ \\
\hline $6: 18$ & $-1,00$ & $-1,00$ & $-1,00$ \\
\hline $12: 12$ & $-0,56$ & $-0,58$ & $-0,23$ \\
\hline $18: 6$ & $+0,68$ & $+0,33$ & $+0,28$ \\
\hline $24: 0$ & $+1,00$ & $+0,75$ & $+1,00$ \\
\hline
\end{tabular}


com sementes e flores ou somente flores (IESF) e mostra que no ensaio de substituição envolvendo Ahv-1:Av-1, em todas as proporções onde entrou Ahv-1, mesmo nas que ela se apresenta em minoria, ela predomina, visto que os valores de IET, IES e IESF variaram de $+0,45$ a $+1,00$.

Do mesmo modo, em Ahv-2:Av-2, considerando IES e IESF, que dão idéia das plantas que estão em fase reprodutiva e, portanto, as maiores responsáveis pela próxima geração, novamente Ahv-2 ganha de Av-2, a partir da proporção 12:12, o mesmo acontecendo com Ahv-2:Ahr-2. Quando se considera Av-2:Ahr-2, o Ahr-2 é que domina sobre o Av-2, ou seja, se estabelece melhor.

De fato, além das suas próprias características, o tamanho e o estádio de desenvolvimento das plantas na vizinhança afetam o desenvolvimento da planta (Grace, 1985; Goldberg, 1990; Caldwell et al., 1996).

De maneira geral, pode-se supor a seguinte seqüência decrescente, em termos de sucesso em competição: A. hybridus - tipo verde $>$ A. hybridus tipo roxo $>A$. viridis; esse sucesso, segundo Thébaud et al. (1996) é determinado pelo balanço das interações positivas e negativas em comunidades vegetais. No entanto, quando, em competição interespecífica, Av-2 entra em maior proporção (proporção 6:18 de Ahv-2:Av-2 e proporção 18:6 de Av-2:Ahr-2) ela domina, uma vez que os índices (IET, IES e IESF) possuem os valores referentes a essa espécie; em qualquer outra proporção, ela não domina. Essa adaptabilidade relativa, conforme a densidade, também foi observada em duas espécies de Avena por Marshall \& Jain (1969).

No caso dos Amaranthus, o Av-2 coabita com Ahv-2 ou Ahr-2 somente se a proporção de sementes de $A$. viridis no solo for maior que a de $A$. hybridus, de forma que $A$. viridis consiga se estabelecer. Esse fato deve estar relacionado, em grande parte, com a capacidade germinativa dessas espécies, pois $A$. viridis tem uma capacidade germinativa inferior à de $A$. hybridus, de forma que ela só se estabelecerá se estiver competindo com maior proporção de sementes, para suprir sua mais baixa germinação (Maluf \& Martins, 1991).

Usando o fogo como fator de estresse em Erica multiflora, Vilá \& Terradas (1995) observaram que tanto o fogo como a vizinhança de plantas adultas reduziram o florescimento e a brotação da espécie; após o incêndio, a regeneração de plantas diminuiu a biomassa de flores, apesar de não ter afetado o vigor do brotamento.

A análise da Tabela 3 mostra que houve um acréscimo na germinação do $10^{\circ}\left(\mathrm{N}_{10}\right)$ ao $20^{\circ}$ dia $\left(\mathrm{N}_{20}\right)$. Porém, na época da colheita, o número total de plantas (NTP) e o número de plantas que entraram em fase reprodutiva (NPS e NPSF) são inferiores ao $\mathrm{N}_{20}$. Deste modo, parece que a germinação tardia vai afetar muito pouco a produção, visto que os valores de NPS e o NPSF estão mais próximos ao $\mathrm{N}_{10}$ que ao $\mathrm{N}_{20}$, o que confirma novamente a idéia de Ross \& Harper (1972) e Harper (1977) de que as sementes que germinam mais rapidamente são as que se estabelecem e, portanto, responsáveis pela geração seguinte.

As análises de variância dos dados de produção mostraram que no ensaio Ahv-1:Av-1 houve diferenças significativas entre as proporções, apenas quanto à produção de folhas $\left(\mathrm{V}_{1}\right)$; as comparações de médias pelo teste de Tukey (Tabela 4) evidenciam que apenas a proporção 0:24 foi inferior às demais. Isso mostra que a presença de Ahv-1, em qualquer proporção, bem como seu estande puro, resultou em uma produção de $\mathrm{V}_{1}$ semelhante; essa produção foi superior ao estande puro de Av-1.

No ensaio Ahv-2:Av-2 (as mesmas populações do caso anterior, coletadas em outro local), os resultados foram um pouco diferentes, mas com a mesma tendência. $\mathrm{O}$ teste $\mathrm{F}$ mostrou diferenças significativas quanto à produção de folhas $\left(\mathrm{V}_{1}\right)$, biomassa reprodutiva $(\mathrm{R})$ e produção total $(\mathrm{PT})$. A comparação das médias pelo teste de Tukey (Tabela 4) mostrou que, novamente, apenas a proporção 0:24 foi inferior às demais quanto $a V_{1}$ e R, e foi inferior a 24:0 com relação a PT; as proporções 0:24 e 24:0 diferiram entre si e não diferiram das demais proporções para $\mathrm{PT}$.

Quanto a Ahv-2:Ahr-2, houve diferenças entre as proporções pelo teste $\mathrm{F}$ em relação a $\mathrm{V}_{1}$ e $\mathrm{R}$, porém o teste Tukey (Tabela 4) mostrou diferenças apenas quanto a $\mathrm{R}$, onde à medida que se aumentava a proporção de Ahv-2, aumentava-se o $\mathrm{R}$, o que indica que este biótipo produz mais biomassa reprodutiva que o Ahr-2.

Considerando-se a combinação Av-2:Ahr-2, a análise de variância mostrou diferenças entre as 
proporções apenas quanto à produção de ramos + caules $\left(\mathrm{V}_{2}\right)$, porém o teste de Tukey, com relação a médias (Tabela 4), não detectou diferenças entre essas proporções, apesar de as médias de $\mathrm{V}_{1}, \mathrm{~V}_{2}, \mathrm{~V}$ e PT mostrarem a tendência de Ahr-2 produzir mais biomassa vegetativa que Av-2, o que indica que Ahr-2 é mais estrategista-K que Av-2.

Este estudo não mostrou a superioridade dos estandes mistos de Amaranthus, quando comparados aos estandes puros. No entanto, estudos anteriores mostraram que os estandes mistos de trigo (Rajeswara Rao \& Prassad, 1984) produziram mais grãos que os estandes puros. Segundo Harper (1968), a superioridade dos estandes mistos - quando comparados aos estandes puros - não implica mutualismo balanceado, mas pode ser explicado em termos da reduzida interferência de uma cultivar na outra, o que deve ter ocorrido no caso dos Amaranthus.

A análise da Fig.1, da produção total relativa (PTR) quanto a $\mathrm{V}_{1}, \mathrm{~V}_{2}, \mathrm{R}$ e PT, mostra que nos ensaios de substituição envolvendo Ahv-1:Av-1 e Ahv-2:Ahr-2, em todas as proporções utilizadas, as produções (PTR) foram praticamente semelhantes (estandes mistos semelhantes aos estandes puros), visto que todos os valores estão próximos a 1 , o que indica que as populações estão demandando recursos semelhantes do ambiente, como postulado por Harper (1977).

Entretanto, no que tange a Ahv-2:Av-2, a resposta foi diferente (Fig.1). Considerando-se

TABELA 3. Número de plantas aos 10 dias $\left(\mathrm{N}_{10}\right)$, aos 20 dias $\left(\mathrm{N}_{20}\right)$, total de plantas (NTP), número de plantas com sementes (NPS) e de plantas com sementes e flores ou somente flores (NPSF), expressos em porcentagem da densidade de semeadura $\left(\mathrm{D}_{0}\right.$ - 24 sementes/vaso), para Amaranthus, em quatro ensaios de substituição independentes, envolvendo as duas populações do local 1 (Ahv-1:Av-1) e as três populações do local 2 (Ahv-2:Av-2; Ahv2:Ahr-2; Av-2:Ahr-2).

\begin{tabular}{|c|c|c|c|c|c|}
\hline Proporções $^{1}$ & $\mathrm{~N}_{10}$ & $\mathrm{~N}_{20}$ & NTP & NPS & NPSF \\
\hline \multicolumn{6}{|l|}{ Ahv-1:Av-1 } \\
\hline $0: 24$ & 23,96 & 66,67 & 45,83 & 23,96 & 27,08 \\
\hline $6: 18$ & 25,00 & 63,54 & 37,50 & 15,62 & 21,87 \\
\hline $12: 12$ & 33,33 & 73,96 & 42,71 & 13,54 & 27,08 \\
\hline $18: 6$ & 34,37 & 77,08 & 60,42 & 17,71 & 27,08 \\
\hline $24: 0$ & 57,29 & 83,33 & 75,00 & 29,17 & 45,83 \\
\hline \multicolumn{6}{|l|}{ Ahv-2:Av-2 } \\
\hline $0: 24$ & 11,46 & 78,12 & 38,54 & 9,38 & 13,54 \\
\hline $6: 18$ & 19,79 & 71,87 & 42,71 & 19,79 & 23,96 \\
\hline $12: 12$ & 28,12 & 60,42 & 40,63 & 17,71 & 31,25 \\
\hline $18: 6$ & 28,12 & 55,21 & 36,46 & 15,62 & 30,21 \\
\hline $24: 0$ & 40,62 & 43,75 & 44,79 & 13,54 & 41,67 \\
\hline \multicolumn{6}{|l|}{ Ahv-2:Ahr-2 } \\
\hline $0: 24$ & 15,62 & 62,50 & 38,54 & 5,21 & 8,33 \\
\hline $6: 18$ & 38,54 & 70,83 & 50,00 & 10,42 & 23,96 \\
\hline $12: 12$ & 37,50 & 53,12 & 35,42 & 12,50 & 23,96 \\
\hline $18: 6$ & 41,67 & 55,21 & 46,87 & 12,50 & 26,04 \\
\hline $24: 0$ & 45,83 & 55,21 & 52,08 & 22,92 & 44,79 \\
\hline \multicolumn{6}{|l|}{ Av-2:Ahr-2 } \\
\hline $0: 24$ & 23,96 & 58,33 & 34,37 & 9,38 & 17,71 \\
\hline $6: 18$ & 12,50 & 52,08 & 23,96 & 7,29 & 8,33 \\
\hline $12: 12$ & 6,25 & 64,58 & 35,42 & 5,21 & 9,38 \\
\hline $18: 6$ & 11,46 & 76,04 & 46,87 & 4,17 & 11,46 \\
\hline $24: 0$ & 4,17 & 72,92 & 29,17 & 5,21 & 8,33 \\
\hline
\end{tabular}

1 Ahv-1: Amaranthus hybridus - tipo verde, do local 1; Av-1: A. viridis, do local 1; Ahv-2: A. hybridus - tipo verde, do local 2; Ahr-2: A. hybridus - tipo roxo, do local 2; Av-2: A. viridis, do local 2. 
TABELA 4. Média de produção de matéria seca, em gramas, para folhas $\left(V_{1}\right)$, caules+ $\operatorname{ramos}\left(V_{2}\right)$, biomassa vegetativa da parte aérea $\left(V=V_{1}+V_{2}\right)$, biomassa reprodutiva $(R)$ e produção total $(P T=V+R)$, para Amaranthus, em quatro ensaios de substituição independentes, envolvendo as duas populações do local 1 (Ahv-1:Av-1) e as três populações do local 2 (Ahv-2:Av-2; Ahv-2:Ahr-2; Av-2:Ahr-2).

\begin{tabular}{|c|c|c|c|c|c|}
\hline Proporções $^{1}$ & $\mathrm{~V}_{1}$ & $\mathrm{~V}_{2}$ & $\mathrm{~V}$ & $\mathrm{R}$ & PT \\
\hline \multicolumn{6}{|l|}{ Ahv-1:Av-1 } \\
\hline $0: 24$ & 1,15 & 3,57 & 4,72 & 1,30 & 6,01 \\
\hline $6: 18$ & 2,24 & 4,04 & 6,28 & 4,30 & 10,58 \\
\hline $12: 12$ & 2,48 & 4,08 & 6,56 & 2,59 & 9,14 \\
\hline $18: 6$ & 2,47 & 3,56 & 6,03 & 2,58 & 8,61 \\
\hline $24: 0$ & 2,90 & 3,83 & 6,74 & 2,79 & 9,52 \\
\hline $\mathrm{DMS}_{5 \%}$ & 0,84 & 1,65 & 2,16 & 3,39 & 4,68 \\
\hline $\mathrm{DMS}_{1 \%}$ & 1,09 & 2,14 & 2,80 & 4,40 & 6,06 \\
\hline CV (\%) & 16,63 & 19,21 & 15,79 & 55,55 & 23,67 \\
\hline \multicolumn{6}{|l|}{ Ahv-2:Av-2 } \\
\hline $0: 24$ & 0,66 & 1,66 & 2,32 & 0,54 & 2,87 \\
\hline $6: 18$ & 1,77 & 2,87 & 4,64 & 2,00 & 2,63 \\
\hline $12: 12$ & 2,03 & 2,52 & 4,54 & 1,06 & 6,40 \\
\hline $18: 6$ & 1,87 & 2,17 & 4,04 & 2,04 & 6,08 \\
\hline $24: 0$ & 2,28 & 3,32 & 5,61 & 2,37 & 7,98 \\
\hline $\mathrm{DMS}_{5 \%}$ & 0,58 & 3,02 & 3,40 & 0,80 & 3,04 \\
\hline $\mathrm{DMS}_{1 \%}$ & 0,75 & 3,91 & 4,40 & 1,04 & 4,97 \\
\hline CV (\%) & 15,00 & 53,36 & 35,67 & 20,25 & 28,43 \\
\hline \multicolumn{6}{|l|}{ Ahv-2:Ahr-2 } \\
\hline $0: 24$ & 1,83 & 3,42 & 5,25 & 1,02 & 6,27 \\
\hline $6: 18$ & 2,25 & 3,63 & 5,88 & 1,36 & 7,24 \\
\hline $12: 12$ & 2,06 & 4,12 & 6,18 & 1,44 & 7,62 \\
\hline $18: 6$ & 1,87 & 3,38 & 5,25 & 1,99 & 7,24 \\
\hline $24: 0$ & 2,52 & 3,34 & 6,16 & 2,31 & 8,47 \\
\hline $\mathrm{DMS}_{5 \%}$ & 0,70 & 1,52 & 2,05 & 0,81 & 2,65 \\
\hline $\mathrm{DMS}_{1 \%}$ & 0,90 & 1,96 & 2,66 & 1,05 & 3,43 \\
\hline CV (\%) & 14,62 & 18,46 & 15,84 & 22,28 & 15,93 \\
\hline \multicolumn{6}{|l|}{ Av-2:Ahr-2 } \\
\hline $0: 24$ & 3,06 & 4,08 & 7,14 & 1,28 & 8,42 \\
\hline $6: 18$ & 3,47 & 5,25 & 8,72 & 2,30 & 11,03 \\
\hline $12: 12$ & 3,09 & 4,36 & 7,45 & 1,29 & 8,74 \\
\hline $18: 6$ & 1,80 & 2,04 & 3,83 & 0,70 & 4,53 \\
\hline 24:0 & 1,05 & 1,33 & 2,38 & 1,23 & 3,61 \\
\hline $\mathrm{DMS}_{5 \%}$ & 2,97 & 3,98 & 6,84 & 2,16 & 8,63 \\
\hline $\mathrm{DMS}_{1 \%}$ & 3,85 & 5,15 & 8,86 & 2,80 & 11,18 \\
\hline CV (\%) & 52,95 & 51,76 & 51,44 & 70,46 & 52,65 \\
\hline
\end{tabular}

${ }^{1} \mathrm{DMS}_{5 \%}$ : diferença mínima significativa pelo teste de Tukey, a 5\% de probabilidade; $\mathrm{DMS}_{1 \%}$ : diferença mínima significativa pelo teste de Tukey, a $1 \%$ de probabilidade; Ahv-1: Amaranthus hybridus - tipo verde, do local 1; Av-1: A. viridis, do local 1; Ahv-2: A. hybridus - tipo verde, do local 2; Ahr-2: A. hybridus - tipo roxo, do local 2; Av-2: A. viridis, do local 2. 
todas as características, nas proporções 6:18 e 12:12, parece que as populações estão utilizando recursos diferentes do ambiente (PTR $>1$ ), evitando competição (Harper, 1977). Somente na proporção 18:6 é que elas competem entre si (PTR próximo a 1).

Analisando-se o ensaio Av-2:Ahr-2, a respeito de todas as características, na proporção 18:6, parece existir antagonismo mútuo entre as espécies $($ PTR $<1)$. Nesse caso, quando se considera a produção isolada de biomassa reprodutiva $(\mathrm{R})$, ocorreu uma resposta muito diferente das outras características $\left(\mathrm{V}_{1}, \mathrm{~V}_{2}\right.$ e PT) na proporção 6:18, que talvez possa ser interpretada como as populações estarem demandando os mesmos recursos para
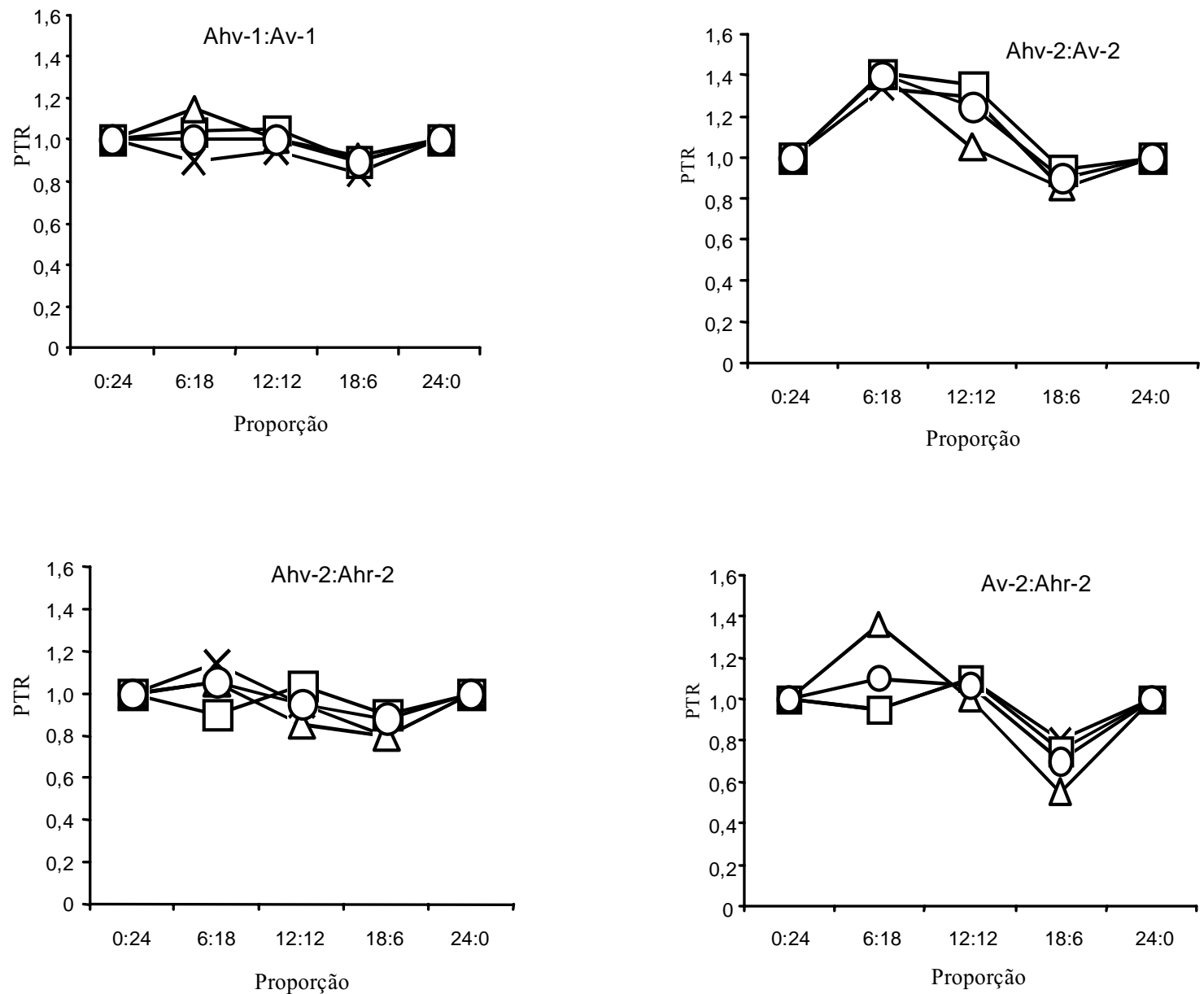

FIG. 1. Produção total relativa (PTR) de folhas $\left(-X-V_{1}\right)$, ramos + caules $\left(--V_{2}\right)$, biomassa reprodutiva (- $\Delta-$ R) e produção total (-O- PT) de Amaranthus, em quatro ensaios de substituição independentes, envolvendo as duas populações do local 1 (Ahv-1:Av-1) e as três populações do local 2 (Ahv-2:Av-2; Ahv-2:Ahr-2; Av-2:Ahr-2). Ahv-1: Amaranthus hybridus - tipo verde, do local 1; Av-1: A. viridis, do local 1; Ahv-2: A. hybridus - tipo verde, do local 2; Ahr-2: A. hybridus - tipo roxo, do local 2; Av-2: A. viridis, do local 2. 
formação de biomassa vegetativa e utilizando estratégias metabólicas diferentes de translocação de nutrientes das estruturas vegetativas para as de reprodução, como em todos os casos já discutidos em que PTR $>1$.

As análises de correlação entre caracteres (Tabela 5) mostram que, de modo geral, os números de plantas na época do desmonte das parcelas (NTP, NPS e NPSF) têm maior correlação com o número de plantas estabelecidas aos 10 dias $\left(\mathrm{N}_{10}\right)$, do que aos 20 dias $\left(\mathrm{N}_{20}\right)$, o que evidencia novamente que as sementes que germinam rapidamente são as responsáveis pela próxima geração.

TABELA 5. Correlações simples entre número de plantas aos 10 dias $\left(\mathrm{N}_{10}\right)$ e aos 20 dias $\left(\mathrm{N}_{20}\right)$ com número total de plantas (NTP), número de plantas com sementes (NPS) e número de plantas com sementes e flores ou somente flores, em quatro ensaios de substituição independente, envolvendo as duas populações do local 1 (Ahv-1:Av-1) e as três populações do local 2 (Ahv-2:Av-2; Ahv-2:Ahr-2; Av-2:Ahr-2) ${ }^{1}$.

\begin{tabular}{lccc}
\hline Correlações & NTP & NPS & NPSF \\
\hline \multicolumn{3}{c}{ Ahv-1:Av-1 } \\
$\mathrm{N}_{10}$ & $0,77^{* *}$ & $0,72^{* *}$ & $0,85^{* *}$ \\
$\mathrm{~N}_{20}$ & $0,64^{* *}$ & $0,39^{\text {ns }}$ & $0,45^{*}$ \\
& \multicolumn{3}{c}{ Ahv-2:Av-2 } \\
$\mathrm{N}_{10}$ & $0,42^{\text {ns }}$ & $0,52^{*}$ & $0,90^{* *}$ \\
$\mathrm{~N}_{20}$ & $0,33^{\text {ns }}$ & $0,17^{\text {ns }}$ & $-0,27^{\text {ns }}$ \\
& \multicolumn{3}{c}{ Ahv-2:Ahr-2 } \\
$\mathrm{N}_{10}$ & $0,75^{* *}$ & $0,66^{* *}$ & $0,73^{* *}$ \\
$\mathrm{~N}_{20}$ & $0,53^{*}$ & $-0,07^{\text {ns }}$ & $-0,04^{\text {ns }}$ \\
& \multicolumn{3}{c}{ Av- $2: A h r-2$} \\
$\mathrm{~N}_{10}$ & $0,20^{\text {ns }}$ & $0,72^{* *}$ & $0,83^{* *}$ \\
$\mathrm{~N}_{20}$ & $0,57^{*}$ & $-0,23^{\text {ns }}$ & $-0,01^{\text {ns }}$ \\
\hline
\end{tabular}

1 Ahv-1: Amaranthus hybridus - tipo verde, do local 1; Av-1: A. viridis, do local 1; Ahv-2: A. hybridus - tipo verde, do local 2; Ahr-2: A. hybridus - tipo roxo, do local 2; Av-2: A. viridis, do local 2.

ns, $*$ e ** Não-significativo e significativo a $5 \%$ e $1 \%$ de probabilidade, respectivamente.

\section{CONCLUSÕES}

1. As populações que germinam mais prontamente são as que vencem em competição, ou seja, se estabelecem, e produzem maior número de sementes.

2. Em condições competitivas, há tendência de Amaranthus hybridus (tipo verde) dominar tanto $A$. hybridus (tipo roxo) como $A$. viridis, e de A. hybridus (tipo roxo) dominar $A$. viridis.

\section{REFERÊNCIAS}

BLACK, C.C.; CHEN, T.M.; BROWN, R.H. Biochemical basis for plant competition. Weed Science, Ithaca, v.17, p.338-344, 1969.

CALDWELL, M.M.; MANWARING, J.M.; DURHAM, S.L. Species interations at the level of fine roots in the field influence of soil nutrient heterogeneity and plant size. Oecologia, Berlin, v.106, p.440-447, 1996.

CALLAWAY, R.M.; WALKER, L.R. Competition and facilitation: a synthetic approach to interactions in plant communities. Ecology, Brooklyn, v.78, p.1958-1965, 1997.

DeWIT, C.T.; VAN DEN BERGH, J.P. Competition between herbage plants. Netherlands Journal of Agricultural Science, Wageningen, v.13, p.213-221, 1965.

GOLDBERG, D.E. Components of resource competition in plant comunities. In: GRACE, J.; TILMAN, D. (Eds.). Perspectives on plant competition. New York: Academic Press, 1990. p.27-49.

GRACE, J.B. Juvenile vs. adult competitive abilities in plants: size-dependence in cattails (Typha). Ecology, Brooklyn, v.66, p.1630-1638, 1985.

HARPER, J.L. Population biology of plants. London: Academic Press, 1977. 892p.

HARPER, J.L. The regulation of numbers and mass in plant populations. In: LEWONTIN, R.C. (Ed.). Population biology and evolution. Syracuse: Syracuse University Press, 1968. p.139-158.

HARPER, J.L.; OGDEN, J. Reproductive strategy of heigher plants; the concept of strategy with special reference to Senecio vulgaris L. Journal of Ecology, London, v.58, p.681-698, 1970.

Pesq. agropec. bras., Brasília, v.34, n.5, p.723-732, maio 1999 
HOLMGREN, M.; SCHEFFER, M.; HUSTON, M.A. The interplay of facilitation and competition in plant communities. Ecology, Brooklyn, v.78, p.19661975, 1997.

MALUF, A.M.; MARTINS, P.S. Germinação de sementes de Amaranthus hybridus L. e A. viridis L. Revista Brasileira de Biologia, v.5, n.2, p.417-425, 1991.

MARSHALL, D.R.; JAIN, S.K. Interference in pure and mixed populations of Avena fatua and A. barbata. Journal of Ecology, London, v.57, p.251-270, 1969.

McNAUGHTON, S.J.; WOLF, L.L. General ecology. New York: Holt, Rinehart and Winston, 1973. 710p.

PIMENTEL-GOMES, F. Curso de estatística experimental. São Paulo: Nobel, 1981. 430p.

RAJESWARA RAO, B.R.; PRASSAD, R. Intergenotype competition in mixed stands of spring wheat genotypes. Euphytica, Wageningen, v.33, p.241-247, 1984.

ROSS, M.A.; HARPER, J.L. Occupation of biological space during seedling establishment. Journal of Ecology, London, v.60, p.77-88, 1972.
SCHREIBER, M.M. A technique for studying weed competition in forage legume establishment. Weeds, Ithaca, v.15, p.1-4, 1967.

STEEL, R.G.D.; TORRIE, J.H. Principles and procedures of statistics. New York: McGraw-Hill, 1980. 481p.

THÉBAUD, C.; FINZE, A.C.; AFFRE, L.; DEBUSSCHE, M.; ESCARRÉ, J. Assessing why two introduced Conyza differ in their ability to invade mediterranean old fields. Ecology, Brooklyn, v.78, p.1958-1965, 1996.

VARGAS, M.F.P. Ecologia de populações de espécies de Amaranthus L., que ocorrem no Estado de São Paulo, Brasil. Piracicaba: USP- ESALQ, 1985.99p. Dissertação de Mestrado.

VILÁ, M.; TERRADAS, J. Effects on competition and disturbance on the resprouting performance of the Mediterranean shrub Erica multiflora L. (Ericaceae). American Journal of Botany, v.82, n.10, p.1241-1248, 1995. 